Dialectologia 16 (2016), 93-116.

ISSN: 2013-2247

Received 10 August 2014.

Accepted 2 December 2014.

\title{
EL NIVEL SOCIAL COMO INDICADOR DE LA VARIACIÓN DE -/S/ EN EL HABLA DE MÉRIDA (BADAJOZ)
}

\author{
Elena Fernández de Molina Ortés \\ Universidad de Burgos* \\ efortes@ub.es
}

Resumen

En esta investigación realizamos un análisis sobre las diferentes realizaciones del sonido -/s/ en posición implosiva en la localidad extremeña de Mérida. Para ello, utilizaremos los datos obtenidos de las encuestas y entrevistas realizados en el municipio para comprobar, primero, si los factores lingüísticos (distribucionales y contextuales) influyen en el uso de las diferentes realizaciones de -/s/ en Mérida pero, sobre todo, para advertir si el nivel social, teniendo en cuenta el nivel de instrucción de los informantes, incide en la selección de las diferentes variantes fonéticas seleccionadas en el municipio.

\section{Palabras clave}

fonética, fricativa -/s/, variación, Mérida, extremeño

THE SOCIAL STATUS AS AN INDICATOR OF THE VARIATION OF - /s/ IN SPEECH OF MERIDA (BADAJOZ) Abstract

In this paper we present an analysis of different variants of sound $-/ \mathrm{s} /$ in implosive position in the town of Mérida (Badajoz). To do this, we use data from surveys and interviews conducted in the town to see if linguistic factors (distributional and contextual) are influential in the selection of variants of -/s/; also, specially, we will use this information to observe if the social status (depending on the level of education of the speakers) affects the selection of the different phonetic variants selected in the township.

* Facultad de Humanidades y Comunicación. Departamento de Filología. c/Villadiego s/n 09001 Burgos. 


\section{Keywords}

phonetic, fricative -/s/, variation, Mérida, extremenian

\section{Introducción}

La variación del fonema sibilante, fricativo, sordo /s/ en posición implosiva ha sido un fenómeno analizado en diversos estudios de sociolingüística y dialectología hispánica tanto de España (Moya 1979; Samper 1999; Calero 1993; Blanco 1997; Molina 1998; Vida 2003) como de Hispanoamérica (Cedergren 1983; Terrell 1978; López Morales 1983). Como bien afirma Samper Padilla (2001), el sonido cumple las características que debe tener un fenómeno para ser objeto de estudio: por una parte, es un elemento de elevada frecuencia en el discurso que está muy integrado en el sistema pero, además, tiene una estratificación asimétrica que permite que el individuo, al usarlo en diferentes contextos, no sea consciente de su pronunciación.

Si consideramos, con Dámaso Alonso, que la -/s/ implosiva "es lo mismo que hablar del grupo s+ consonante, porque tal grupo existe: a) en posición interior de palabra, b) cuando la -s final de palabra sigue, sin pausa, otra voz que comienza por consonante" (Alonso 1973: 75) y comprobamos los resultados de las investigaciones realizadas hasta la actualidad, podemos advertir que este sonido ha sufrido, en amplias zonas del dominio hispánico, un proceso de debilitamiento que comienza con la aspiración de -/s/ y que, en algunos casos, concluye con la elisión total del segmento. No obstante, también existen ciertos factores lingüísticos, sociales y geográficos que, como veremos, influyen en sus diversas realizaciones.

Con esta investigación pretendemos contribuir al estudio de la variación de -/s/ en España pero, también, ampliar las monografías sobre la variación lingüística de un área concreta del país, Extremadura, analizando el habla de Mérida, localidad que forma parte de la provincia de Badajoz y que, desde el año 1983, es capital autonómica de la región. Aunque en este análisis únicamente se estudiará un rasgo fonético concreto, es necesario advertir que este forma parte de una investigación mucho más amplia, el estudio sobre El habla de Mérida, que nació como actualización de la obra realizada por don Alonso Zamora Vicente sobre El habla de Mérida y sus cercanías (Zamora Vicente 1943) y en la que se representan los cambios lingüísticos que ha 
sufrido la actual capital de Extremadura desde los años cuarenta del siglo pasado hasta las primeras décadas del siglo XXI.

Para este estudio tendremos en cuenta algunos factores lingüísticos como el lugar que ocupa el sonido en la palabra o el contexto en el que está situado; no obstante indicaremos, de igual forma, la influencia de los factores sociales atendiendo, fundamentalmente, al nivel de instrucción de los hablantes de la localidad y será preciso, además, recurrir a la situación geográfica en la que se encuentra el municipio estudiado para la selección de las variantes de la fricativa. Todas estas consideraciones podrán advertirse en apartados posteriores.

\section{La distribución de -/s/ en el mundo hispánico. Delimitación geográfica y factores del cambio}

Como afirma Martín Butragueño (1995: 7), el estudio de la -/s/ ha permitido delimitar la segmentación geolingüística del español centro-septentrional y meridional de la Península. ${ }^{1}$ Tradicionalmente, se ha asegurado que la aspiración y la elisión de la fricativa eran realizaciones circunscritas a las hablas andaluzas (sobre todo, en la zona centro-occidental de la región) aunque, tal como han demostrado investigaciones posteriores, estas afirmaciones son discutibles. Primeramente, según los datos que ofrece el $A L P I$, la aspiración sobrepasa la región andaluza y aparece en Extremadura, Murcia, Albacete y Toledo y rebasa la zona meridional de Salamanca, Guadalajara, Cuenca, Ávila, Toledo y Madrid (Alonso 1973: 76; Zamora Vicente 1979: 71; Fernández Sevilla 1980: 475). ${ }^{2}$ Incluso en fechas recientes, autores como García Mouton \& Moreno Fernández (1994: 127) han comprobado que la aspiración de -/s/ aparece más al norte de lo que representó el $A L P I$, en zonas como Santander.

\footnotetext{
${ }^{1}$ Martín Butragueño (1995: 7) afirma, incluso, que ambas realizaciones representan dos zonas peninsulares: las zonas de tendencia conservadora, en las que se mantiene el sonido fricativo y que representan el llamado español atlántico y las zonas de tendencia evolutiva, donde se advierten las variantes aspiradas y elididas del sonido.

2 Las diferentes variantes de -/s/ se han podido comprobar en los mapas 17 (“árboles"), 19 ("avispa”), 65 (“desbocado"), 66 (“desnudo") y 72 (“los domingos").
} 
Tal como hemos advertido en líneas anteriores, en este estudio comprobaremos las diferentes variantes de $-/ \mathrm{s} /$ en el habla de Mérida, localidad situada en Extremadura. Si tenemos en cuenta las afirmaciones que realizó Zamora Vicente en el estudio de la localidad en los años cuarenta del siglo pasado, la aspiración de $-/ \mathrm{s} / \mathrm{se}$ advertía, como variante sistemática, tanto en posición final de grupo como de sílaba aunque, como precisó el autor, su articulación estaba motivada por los sonidos cercanos. No obstante, don Alonso afirmó que, en posición final absoluta, la -/s/ "se pierde casi totalmente. Desde luego, el efecto acústico no existe" (Zamora Vicente 1943: 23).

Además, para observar cuál es la situación lingüística de este sonido en la región tendremos en cuenta los datos que González Salgado ofrece en la Cartografía Lingüística de Extremadura (González Salgado 2003a); ${ }^{3}$ según los resultados obtenidos en su investigación, el autor afirma que la aspiración de -/s/ aparece en las 74 localidades estudiadas excepto en Eljas, Cedillo y Olivenza zonas influidas, notablemente, por el portugués (González Salgado 2003b: 599). Al igual que Zamora Vicente, González Salgado observa que, en la región, el sonido se mantiene cuando aparece seguido de vocal aunque, incluso en este contexto, se pueden encontrar casos de aspiración ${ }^{4}$.

La extensión geográfica de las variantes aspiradas y elididas de -/s/ que, tal como se ha podido comprobar en párrafos anteriores, es común en una zona amplísima de la Península, representa la evolución de un proceso de debilitamiento que, aunque como afirmó Fernández Sevilla (1980), ha intentado suavizarse desde las últimas décadas por factores como la presión cultista, en la actualidad se mantiene en un estado latente (Fernández Sevilla 1980: 487) ${ }^{5}$.

\footnotetext{
${ }^{3}$ Ante la esperada llegada del Atlas Lingüístico y Etnográfico de Extremadura, estos materiales permiten, en los estudios sobre las hablas extremeñas, comprobar los resultados lingüísticos de la región y tener una perspectiva amplia de rasgos fonéticos, morfosintácticos y léxicos de 74 localidades, 35 de Cáceres y 36 de Badajoz e, incluso, 3 del norte de Andalucía occidental.

${ }^{4}$ Otros estudios sobre el habla en Extremadura como los realizados por Cummins (1974) sobre el habla de Coria o Montero Curiel (1997) sobre Madroñera advierten que, aunque la aspiración es general en ambas localidades, en el contexto -s+vocal la fricativa suele mantenerse.

${ }^{5}$ Navarro Tomás consideró vulgarismo "la pronunciación de la $s$ final como una simple aspiración, y asimismo su eliminación total en determinadas circunstancias [y] hechos corrientes (Navarro Tomás 2004: 110).
} 
Este proceso de relajación del sonido al que nos referimos está producido, entre otras cosas, como consecuencia del modo de hablar natural, rápido y descuidado (Widdison 1993) y, aunque es evidente que la procedencia geográfica del hablante es un componente esencial, para comprobar la extensión del debilitamiento de $-/ \mathrm{s} /$ existen, de igual forma, factores lingüísticos que influyen en el cambio de articulación de la fricativa en según qué contextos.

Por una parte, en su estudio sobre la -/s/ en el español Cubano, Terrell (1979) propuso que el proceso está motivado por la posición de la fricativa en la palabra; de esta forma, según el autor, el proceso debió iniciarse en interior de palabra y, en un segundo momento, pasó a la posición final, ante consonante. ${ }^{6}$ Méndez Dosuna (1987), en cambio, afirmaba que la variación de -/s/ estaba influida por el contacto de sílabas; según el principio de silabación preferida, en español sería inaceptable el uso de $s$ ante semiconsonantes, líquidas, nasales o fricativas sonoras por lo que, en este caso, la /s/ ocuparía un grado mayor de fuerza de la consonante inicial y provocaría reajustes fonéticos como la aspiración de la implosiva. ${ }^{7}$

Todos estos factores, lingüísticos y geográficos, influyen de forma evidente en el mantenimiento o no de la variante estándar, fricativa y sorda -/s/. En esta investigación tendremos en cuenta, como bien se expuso en el apartado introductorio de este trabajo estos factores pero, además, estudiaremos si existen causas sociales que determinan el uso de las variantes del sonido.

\footnotetext{
${ }^{6}$ Terrell advirtió que, ante vocal, el fenómeno se produce por la generalización formal de contextos en un orden de simplificación decreciente que aparece, primero, ante consonante, después ante consonante y vocal y, finalmente, ante consonante, vocal y pausa.

${ }^{7}$ Además de los factores fonéticos, la presencia de diferentes variantes de -/s/ producida por la función gramatical de la fricativa, bien como marca de plural o bien como segunda persona del singular del verbo, ha sido objeto de estudio en diversas investigaciones sobre el español de España y de América. Según la hipótesis funcional de Kiparsky (1983), en aquellos casos en los que la -/s/ es indispensable para no entorpecer la llegada del mensaje comunicativo, desde el punto de vista gramatical, los hablantes mantienen el sonido como marca no redundante. Autores como Labov (1972) o Herasimchuck (1972), entre otros, pudieron confirmar esta propuesta aunque, tal como se ha podido comprobar en los últimos años, el factor funcional no parece ser relevante para conocer la realidad del fenómeno (Alvar 1972; Almeida 1989; Molina Martos 1991; Samper 1999).
} 


\section{Metodología para la obtención de resultados}

Para esta investigación sobre la variación de $-/ \mathrm{s} /$ en el habla de Mérida se utilizaron los mismos procedimientos empleados para el estudio del habla de la localidad. Por ello, a continuación se presentarán, de la forma más exhaustiva posible, las pautas que se siguieron para la obtención de la muestra de la población, la selección de variables sociales y la elección de variantes lingüísticas.

\subsection{Selección de variables extralingüisticas: el nivel social}

En este estudio se tendrá en consideración, únicamente, el nivel social de los informantes para advertir, en los análisis propuestos, cómo este factor influye en el uso de cada una de las variantes lingüísticas propuestas para el estudio de $-/ \mathrm{s} /$ en posición implosiva.

En esta investigación se usó un único indicador perteneciente al modelo de estratificación social: ${ }^{8}$ el nivel de instrucción. ${ }^{9}$ En Mérida se distinguieron tres niveles sociales atendiendo al tiempo de formación del individuo: en el nivel bajo, por una parte, se incluyeron aquellos habitantes que no saben leer ni escribir o que, únicamente, han cursado los estudios primarios, los obligatorios (Bachiller Elemental, Educación Secundaria Obligatoria) o los de Formación Profesional de Grado Medio. Por otra parte, el nivel medio estuvo compuesto por aquellos individuos que han superado la formación de segundo ciclo (Bachiller Superior, COU y Bachillerato) y por aquellos que han realizado un ciclo de Formación Profesional de Grado Superior. Por último, el nivel alto estuvo integrado por los informantes que han superado los estudios universitarios y por aquellos que, además, poseen el título de doctor.

\footnotetext{
${ }^{8}$ Este tipo de modelo procede de la sociología y define a un tipo de sociedad ordenada por capas o estratos; los individuos de los niveles más altos de la sociedad tienen más poder y riqueza frente a los de las capas o estratos inferiores (Trudgill \& Hernández Campoy 2007: 141).

${ }^{9}$ No obstante, la formación académica de los individuos está directamente relacionada con otro nivel, en este caso, el profesional. Evidentemente, el nivel de educación de un individuo influye en la profesión que tiene o puede tener tras su etapa de formación. Por esta razón, aunque en los párrafos posteriores no serán detallados los niveles profesionales que se distinguieron para el estudio del habla de Mérida, hay que advertir que, en el estudio previo, sí se utilizaron tres grupos que se relacionan, directamente, con los tres niveles sociales utilizados para esta investigación.
} 


\subsection{La muestra}

La selección de habitantes para el estudio lingüístico de una población ha sido un problema muy discutido por la sociolingüística; la muestra es una proporción que se presupone representativa de la población y que define la parte del universo que se estima oportuna para el estudio del habla una comunidad. No obstante, y como afirma Moreno Fernández (1990: 69) ${ }^{10}$, "la cantidad ha de responder a los intereses y objetivos del estudio".

El municipio de Mérida estaba compuesto, en el año 2010, por un total de 57.127 habitantes, según los datos del padrón municipal que ofrece el Instituto Nacional de Estadística en su plataforma electrónica. Sin embargo, para realizar el estudio de la localidad se excluyeron, primeramente, los individuos menores de veinte años que componían, en estas fechas, el $23,10 \%$ de la ciudad y el tamaño de la población se redujo a 43.926 habitantes. Además, para limitar de nuevo la extensión de los habitantes de Mérida, únicamente fueron seleccionados aquellos que habían nacido y residían en el municipio pues, de esta forma, podríamos obtener el habla vernácula de la localidad eliminando, de este modo, aquellos habitantes que habían trasladado su lugar de residencia en fechas recientes al municipio y que, por tanto, no representaban sus características lingüísticas. Según estos datos, la población seleccionada estuvo compuesta por un total de 24.887 habitantes.

Teniendo en cuenta estos datos y según el objetivo de esta investigación, para realizar un estudio sobre cómo el nivel social influye en el uso de las diferentes variantes de $-/ \mathrm{s} /$ en el habla de la localidad presentaremos, a continuación, los datos demográficos del municipio atendiendo a los tres niveles anteriormente indicados: bajo, medio y alto, seleccionados según el nivel de instrucción de los habitantes de la localidad.

\footnotetext{
${ }^{10}$ Cuando Labov realizó su estudio sobre el habla de Nueva York (1967) propuso, para la obtención de la muestra de la población, una proporción de hablantes que representara el 1:2500 partes del universo estudiado teniendo en cuenta que, con esta selección, se cumplía el concepto de homogeneidad de la conducta lingüística según el cual, la conducta de un individuo es suficientemente homogénea como para representar, con sus rasgos lingüísticos, un grupo social.
} 


\begin{tabular}{|lcc|}
\hline Bajo & $\mathrm{N}$ & $\%$ \\
\hline Medio & 8835 & 35,50 \\
\hline Alto & 11993 & 40,19 \\
\hline
\end{tabular}

Tabla 1. Distribución demográfica de los habitantes de Mérida según su nivel social.

Una vez obtenidos los datos demográficos de este grupo de la localidad, procedimos a la selección de la muestra sociolingüística para conocer cuántos habitantes eran necesarios para obtener resultados significativos en nuestro estudio. Para ello, utilizamos un tipo de muestreo selectivo por cuotas de afijación proporcional que nos permitió, en este caso, seleccionar el número de individuos que componía cada celda según el peso real que estos tenían en la sociedad. ${ }^{11}$ Por esta razón, se aplicó la fórmula para la obtención de la muestra de poblaciones finitas en las que, $a$ priori, conocemos el número de habitantes objeto de estudio $(n=N$. Z2.p.q /e2 - $(\mathrm{N}-1)$ + Z2.p.q); teniendo en cuenta los datos demográficos anteriormente indicados y según el resultado obtenido de aplicar la fórmula propuesta, el número total de individuos seleccionados en la localidad extremeña fue de 150 habitantes. Según el nivel social de los integrantes de nuestra muestra, el número de informantes por cada celda para el estudio sobre el habla de Mérida es el siguiente:

\begin{tabular}{|l|l|}
\hline Bajo & N \\
\hline Medio & 52 \\
\hline Alto & 73 \\
\hline
\end{tabular}

Tabla 2. Número de habitantes por celda según el nivel social de los habitantes de Mérida.

Evidentemente, los resultados de la Tabla 2 representan los porcentajes de la Tabla 1: en el habla de la localidad serán estudiados más individuos del nivel medio

\footnotetext{
${ }^{11}$ Aunque es cierto que este tipo de muestreo no es habitual en los estudios de sociolingüística actual, es necesario advertir que, evidentemente, cuantos más habitantes sean utilizados para el estudio lingüístico de una comunidad, existe una mayor probabilidad de que los datos obtenidos sean representativos de la misma. No obstante, hasta hoy, el método más utilizado para realizar el muestreo sociolingüístico es el de tipo selectivo cualificado o por cuotas de afijación homogénea, empleado, por ejemplo, para los estudios del grupo PRESEEA en el que cada grupo sociodemográfico aparece representado por el mismo número de individuos.
} 
(73), que componen el $40,19 \%$ del municipio, que de los niveles bajo (53) y alto (25), que representan el $35,50 \%$ y el $16,30 \%$ de la población respectivamente.

\subsection{Métodos de campo}

Los resultados de esta investigación proceden de las entrevistas realizadas a los 150 individuos de la localidad pero, además, de la aplicación de un cuestionario integrado por más de mil preguntas y elaborado (y reelaborado) tomando como modelo el Cuestionario para el Estudio Coordinado para el Estudio de la Norma Culta de España e Hispanoamérica (PILEI 1971). ${ }^{12}$ Por una parte, las entrevistas fueron elaboradas atendiendo al tipo de informantes a las que iban a ser dirigidas; de esta forma, aunque en el inicio de las mismas se propusieron las mismas cuestiones a todos los individuos (datos personales y familiares), posteriormente se trató de complementar la información lingüística que necesitábamos con preguntas sobre los modos de vida o los gustos e intereses de los informantes. El cuestionario, igualmente, se presentó a partir de preguntas dirigidas; estuvo compuesto por doce campos semánticos a partir de los cuales se pudo recopilar el léxico de la localidad pero, además, obtener resultados fonéticos y morfosintácticos.

Los análisis realizados posteriormente para la obtención de las diferentes realizaciones de la -/s/ en posición implosiva fueron posibles gracias a la grabación de los materiales durante la consecución de la recopilación de datos. Fue indispensable tener, como mínimo, media hora de grabación por cada informante seleccionado.

\subsection{Selección de variantes}

Para realizar el estudio de las diferentes realizaciones de la fricativa /s/ en posición implosiva se tendrán en cuenta tres variantes del sonido que serán utilizadas, además, para poder realizar, en análisis posteriores, una comparación con las

\footnotetext{
${ }^{12}$ Solo 95 informantes realizaron el cuestionario completo. No obstante, las entrevistas sí fueron aplicadas a todos los individuos de la localidad.
} 
investigaciones realizadas en las últimas décadas sobre la extensión de las variantes de -/s/ en España e Hispanoamérica ${ }^{13}$.

$\begin{array}{lll}\text { S2 } & {[s]} & \text { sibilante } \\ \text { S1 } & {[\mathrm{h}]} & \text { aspirada } \\ \text { S0 } & {[\varnothing]} & \text { elidida }\end{array}$

La variante S2 representa las realizaciones plenas del sonido, independientemente de su punto de articulación (apical, predorsal, coronal) o el grado de fricción producido por los informantes; por otra parte, en la variante S2 se incluirán aquellos sonidos en los que se ha percibido una aspiración, sorda o sonora. Finalmente, la variante SO representa la ausencia total del fonación en la palabra.

\section{Hipótesis de investigación}

Teniendo en cuenta los datos propuestos en apartados anteriores y atendiendo a las características geográficas y sociolingüísticas que determinan el desarrollo de esta investigación se podría afirmar que, en el habla de Mérida, integrada en las hablas extremeñas, sería habitual encontrar variantes de $-/ \mathrm{s} /$ propias de las hablas meridionales tales como la aspiración o la elisión del sonido.

No obstante, las variantes alternativas al mantenimiento de $-/ \mathrm{s} /$ aparecen, sobre todo, en el habla familiar y en las conversaciones relajadas pero, además, es necesario advertir que su uso implica una desviación de la norma estándar; por esta razón, si tenemos en cuenta los tres niveles sociales seleccionados deberíamos afirmar, en un primer momento, que las realizaciones aspiradas y elididas se encontrarán, de forma más habitual, en los individuos de los niveles sociales bajo y medio y, por último, en los informantes del nivel alto.

\footnotetext{
${ }^{13}$ El uso de estas tres variantes para el estudio del habla de la localidad coincide con el método utilizado por López Morales (1983) para su estudio sobre Puerto Rico así como por Molina Martos (1991) y Calero (1993) sobre Toledo o Samper Padilla (1999) para Las Palmas de Gran Canaria. No obstante, en nuestros análisis no será objeto de estudio la variante asimilada $[\bar{s}]$ porque, aunque se han encontrado resultados aislados, su uso no es representativo en el habla de la localidad.
} 
Finalmente, es importante señalar que existen factores lingüísticos que influyen en el retenimiento o en la selección de variantes lingüísticas alternativas de $-/ \mathrm{s} /$. De esta forma, según los resultados propuestos en diversas investigaciones, en posición final es más frecuente el mantenimiento de la sibilante y la elisión del sonido (Samper 2001); también es cierto que la /s/ en posición final de palabra se conserva mejor ante vocal que ante consonante (Torreblanca 1989: 296) y que fenómenos como la acentuación favorecen el mantenimiento de la variante estándar (S2) (López Morales 1983; Lipski 1983).

Toda estas consideraciones serán tratadas en esta investigación y serán analizadas para comprobar si estas primeras hipótesis pueden ser confirmadas o si, en cambio, los resultados lingüísticos no siguen las tendencias aquí propuestas.

\section{Análisis}

\subsection{Distribución de variantes en Mérida}

Durante la recopilación de datos para el estudio del habla de Mérida se obtuvieron un total de 14525 realizaciones que aparecen distribuidas según las variantes seleccionadas para este análisis de la siguiente forma:

\begin{tabular}{|cc|}
\hline & $\%$ \\
\hline S2 & 8,83 \\
\hline S1 & 69,67 \\
\hline S0 & 21,40 \\
\hline
\end{tabular}

Tabla 3. Distribución de variantes en Mérida.

Como se puede observar en la Tabla 3, la variante habitual en el habla de la localidad es la aspiración, presente en el $69,67 \%$ de las contestaciones de los informantes. También aparece como sonido alternativo la elisión, aunque en un 
número menor de respuestas $(21,40 \%)$ y, por último, se advierten resultados sobre el mantenimiento de la sibilante pero con una frecuencia de uso muy limitada (8,83\%).

Teniendo en cuenta los datos propuestos veamos, a continuación, cómo se distribuyen las diferentes variantes utilizadas para estos análisis según el nivel social de los individuos. De las realizaciones totales del habla de la comunidad, 4678 corresponden al nivel bajo, 6867 al nivel medio y, finalmente, 2867 al nivel alto.

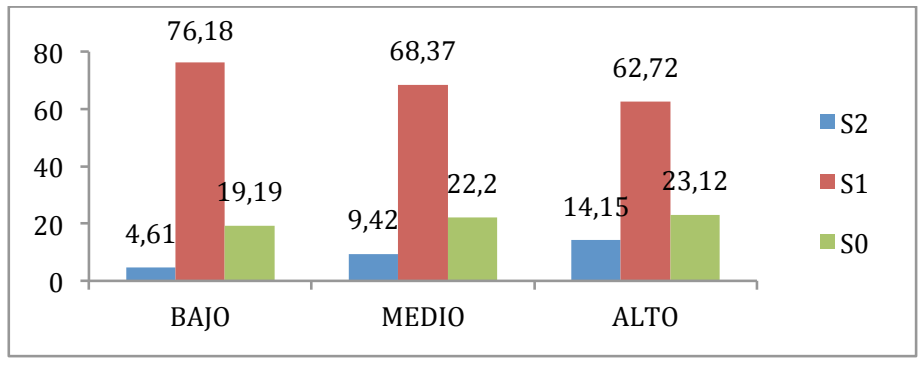

Gráfico 1. Distribución de variantes según el nivel social en Mérida.

Los datos que se presentan en el Gráfico 1 arrojan los resultados obtenidos en los diferentes niveles sociales del habla de Mérida. Como se puede comprobar, el mantenimiento de la variante es mayor cuanto más elevado es el nivel social de los individuos; así, frente al 14,15\% de resultados de S2 en el nivel alto, el uso desciende de forma significativa en el nivel medio, donde se recopilaron únicamente un 9,42\% de resultados del sonido estándar $y$, finalmente, en el nivel bajo, donde la -/s/ se mantuvo en un $4,61 \%$ de las contestaciones.

Por otra parte, aunque como advertimos en la Tabla 3, la variante aspirada es la más habitual en la localidad, el nivel social incide, de nuevo, en la selección de este sonido. De esta forma, a diferencia de los resultados anteriores, cuanto mayor es el nivel social del hablante, menor uso hace de la aspirada; así, frente al 76,18\% de resultados de S2 en las respuestas del nivel bajo, cuanto mayor es el nivel social, menor es el porcentaje de resultados tanto en el nivel medio (68,37\%) como en el nivel alto $(62,72 \%)$.

Finalmente, aun teniendo en cuenta los datos en los corroborábamos las hipótesis propuestas en esta investigación, en el empleo de la variante so comprobamos que el uso la elisión es más común cuanto mayor es el nivel social de los 
individuos; así se ha podido observar en las respuestas del nivel alto $(23,12 \%)$, el nivel medio $(22,2 \%)$ y, por último, el nivel bajo $(19,19 \%)$, donde existe una menor probabilidad de aparición. En este caso, la selección de la variante elidida frente al a estándar o la aspirada se debe, suponemos, a ciertos factores lingüísticos que influyen en la presencia o a la ausencia del sonido y que podremos comprobar, no obstante, en análisis posteriores.

\subsection{Los factores lingüísticos y los factores sociales}

En esta sección comprobaremos si la posición del sonido en la palabra (interior o final) y el contexto en el que este se encuentra (prevocálico y preconsonántico) influyen en el uso de las variantes propuestas para el estudio de la localidad teniendo en cuenta los resultados obtenidos de los niveles sociales seleccionados.

\subsubsection{El factor distribucional y el nivel social}

Como se precisó en apartados anteriores, habitualmente se ha afirmado que la posición interior favorece la aspiración de la -/s/ en posición implosiva (en aquellas zonas en las que aparece la variante) y que el mantenimiento y la elisión del sonido es más común en posición final (Samper 2001).

\begin{tabular}{|ccc|}
\hline & Interior & Final \\
\hline S2 & 5,66 & 13,46 \\
\hline S1 & 94,31 & 31,49 \\
\hline S0 & 0,02 & 54,79 \\
\hline
\end{tabular}

Tabla 4. Distribución de las variantes de -/s/ según su posición.

Según los datos de la Tabla 4, parece que el factor distribucional sí es relevante para conocer la extensión de la variación de -/s/ en la localidad. Si recordamos los resultados de la Tabla 3, en Mérida la aspiración es la solución general pero su uso únicamente es sistemático en posición interior $(94,31 \%)$. En cambio, parece que las 
hipótesis planteadas por Samper se cumplen en el municipio: en posición final, la elisión del sonido suele ser habitual (54,79\%); aunque con menor frecuencia, lo es también la retención del segmento $(13,46 \%)$ frente a la aspiración que, en este caso, parece no ser una variante constante en las respuestas de los informantes $(31,49 \%){ }^{14}$

A partir de estos datos, a continuación comprobaremos si el nivel de los informantes influye en la selección de unas variantes u otras.

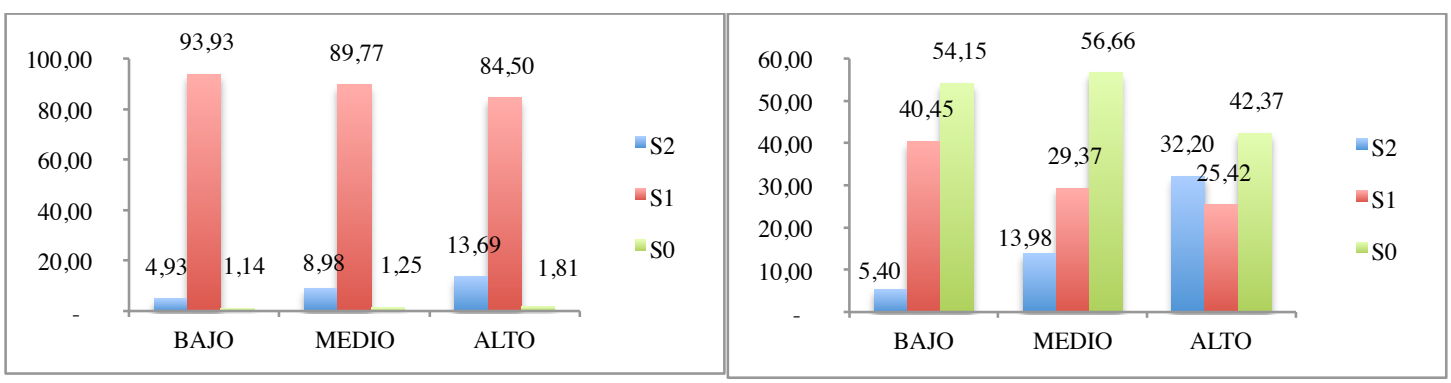

Gráfico 2. Uso de -/s/ en posición interior según

Gráfico 3. Uso de -/s/ en posición final según el nivel el nivel social en Mérida. social en Mérida.

Como se puede observar en el Gráfico 2, la aspiración es la variante habitual en todos los niveles sociales pero, si observamos concretamente los resultados del mantenimiento del sonido (S2), podemos advertir que este aparece, con una mayor frecuencia, en el nivel alto $(13,69 \%)$ y que su uso va descendiendo, progresivamente, cuanto menor es el nivel del individuo.

Si los datos obtenidos en posición interior eran representativos del uso de las variantes teniendo en cuenta el factor extralingüístico, en situación final son aún más significativos. Como se puede apreciar en el Gráfico 3, la única variante con un empleo homogéneo es la elisión; en cambio, el uso de S2 y S1 depende del nivel social de los informantes: el mantenimiento de $-/ \mathrm{s} / \mathrm{es}$, por una parte, considerablemente más elevado en el nivel alto $(32,20 \%)$ frente al nivel medio $(13,97 \%)$ y el bajo $(5,40 \%)$,

\footnotetext{
${ }^{14}$ Los resultados obtenidos en Mérida pueden equipararse a los hallados en otros estudios sobre el segmento -/s/ de España y América. Por una parte, la extensión del uso de la aspiración en posición interior frente a las demás variantes (en este caso, S2 y S0) se advierte en los resultados obtenidos en Alcalá de Henares (Blanco 1997), Las Palmas de Gran Canaria (Samper 1999), Málaga (Vida 2003) o San Juan de Puerto Rico (López Morales 1983) y Buenos Aires (Terrell 1978). En cambio, la preferencia por la elisión del sonido en posición final únicamente se ha encontrado en los estudios sobre Las Palmas (Samper 1999), Málaga (Vida 2003) y Santiago de Los Caballeros (Alba 1982).
} 
donde el porcentaje de uso es muy escaso; en esta posición, los informantes del nivel bajo prefirieron usar la aspiración (40,45 \%) en un mayor número de realizaciones.

Como se ha podido observar en estos análisis, ambos factores, tanto los lingüísticos como los extralingüísticos, son fundamentales para conocer la extensión de uso de las variantes de la localidad.

\subsubsection{El factor contextual y el nivel social}

Teniendo en cuenta que, en posición final, la -/s/ puede aparecer ante vocal, consonante o pausa, en los estudios realizados hasta la actualidad sobre la variación de -/s/ en el mundo hispánico se ha podido comprobar que el sonido suele mantenerse con una mayor frecuencia en el contexto prevocálico frente los segmentos en los que -/s/ se sitúa ante consonante o pausa.

\begin{tabular}{|cccc|}
\hline & $-\mathbf{V}$ & $-\mathbf{C}$ & $-/ /$ \\
\hline S3 & 19,06 & 3,03 & 13,46 \\
\hline S2 & 78,46 & 95,84 & 31,49 \\
\hline S1 & - & - & 0,24 \\
\hline S0 & 2,56 & 1,12 & 54,79 \\
\hline
\end{tabular}

Tabla 5. Distribución de las variantes de -/s/ final de palabra según el contexto fónico.

Estas consideraciones pueden corroborarse en el habla de la localidad; tal como se advierte en los resultados de la Tabla 5 , en el contexto prevocálico la $-/ \mathrm{s} / \mathrm{se}$ mantiene en el 19,06\% de las realizaciones frente en el preconsonántico donde, en cambio, la solución más frecuente es la aspiración $(95,84 \%)$ y, por último, el contexto prepausal en el que la elisión del sonido es la variante más habitual $(54,79 \%) .{ }^{15}$

\footnotetext{
${ }^{15}$ Estos datos corroboran, además, la hipótesis sobre la generalización de contextos que propuso Terrell (1979) en su estudio sobre el español de Cuba; según los datos obtenidos en su investigación, el autor afirmó que, siguiendo un orden intrínseco de las reglas de aspiración y elisión según el contexto en el que se pronuncia el sonido, se puede advertir una correlación sistemática con los contextos fonéticos que se ordenan, inversamente, en dos reglas. De esta forma, si los condicionantes de la aspiración, tal como se ha podido advertir en el habla de Mérida, se dan primero en los contextos preconsonánticos, prevocálicos y prepausales, la regla de elisión debería aparecer (y aparece), primero, ante pausa, después ante vocal y, finalmente, ante consonante. Este esquema pudo confirmarse, de igual forma, en el estudio de López Morales sobre San Juan de Puerto Rico (1983) y en realizado por Cedergren para Panamá (1983).
} 
Hemos comprobado, por tanto, que el factor contextual es, al igual que el distribucional, fundamental para conocer en qué situaciones los informantes de Mérida usan cada una de las variantes. Ahora bien, ¿influye, también, el factor social en esta selección?

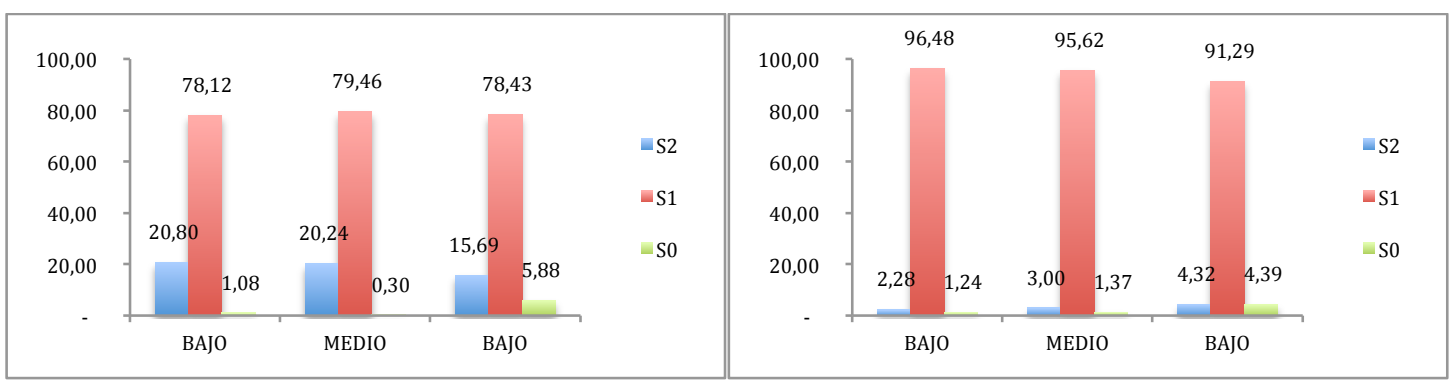

Gráfico 4. Uso de -/s/ en el contexto prevocálico según el nivel social.

Gráfico 5. Uso de -/s/ en el contexto preconsonántico según el nivel social.

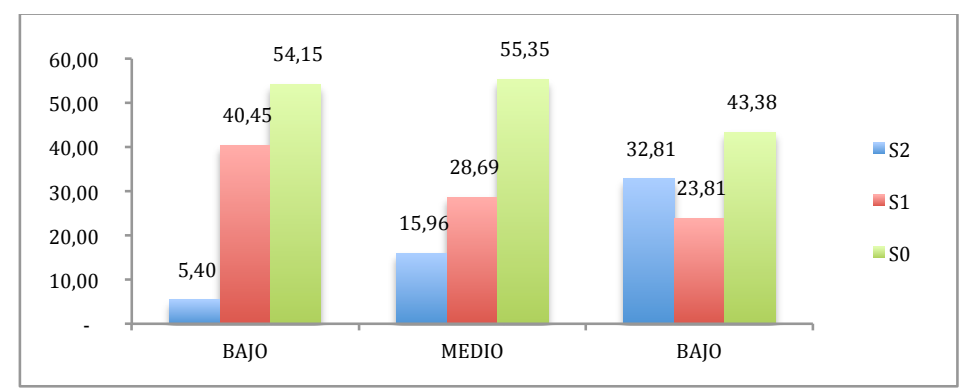

Gráfico 6. Uso de -/s/ en el contexto prepausal según el nivel social.

En este caso, únicamente nos centraremos en los resultados del Gráfico 6 donde se observa que, en el contexto prepausal (en el que predominaba la elisión del sonido), los informantes del nivel alto usaron, con una frecuencia elevada $(32,81 \%)$, la variante S2, frente a los individuos del nivel medio, que la seleccionaron en la mitad de las realizaciones $(15,96 \%)$ y los del nivel bajo, en los que únicamente se percibió la variante estándar en un $5,40 \%$ de las respuestas. En cambio, en este grupo sí predominó la aspiración (40,45\%) que fue la variante alternativa a la elisión.

Por tanto, en el contexto prepausal, el uso de las variantes tiene que ver con el contexto del segmento pero, también, con el nivel social del individuo. 
a) El contexto prevocálico

Aunque, como se ha comprobado en la sección anterior, el contexto prevocálico favorece la retención del sonido, algunos autores (López Morales 1983; Lipski 1983; Samper Padilla 1999) han afirmado que la acentuación de la vocal puede ser, de igual forma, influyente en la retención de $-/ \mathrm{s} /$.

\begin{tabular}{|ccc|} 
& $\ldots$ & \\
\hline S3 & $4,70 \%$ & $44,20 \%$ \\
\hline S2 & $92,06 \%$ & $53,36 \%$ \\
\hline S0 & $3,23 \%$ & $1,42 \%$ \\
\hline
\end{tabular}

Tabla 6. Distribución de las variantes de -/s/ según el contexto prevocálico

De esta forma, si observamos los datos obtenidos en Mérida en la Tabla 6 podemos comprobar que, evidentemente, la variante S2 se mantiene con una frecuencia elevada cuando esta aparece ante vocal tónica $(44,20 \%)$ alternando, de este modo, con la variante local (S2), que aparece en un $53,36 \%$ de las contestaciones. En cambio, cuando la vocal siguiente es átona, los informantes usan la aspiración de forma general.

Por otra parte, si comparamos ahora los datos obtenidos en el habla de la localidad con la variación del sonido según el nivel social de los informantes, podremos advertir resultados reveladores:

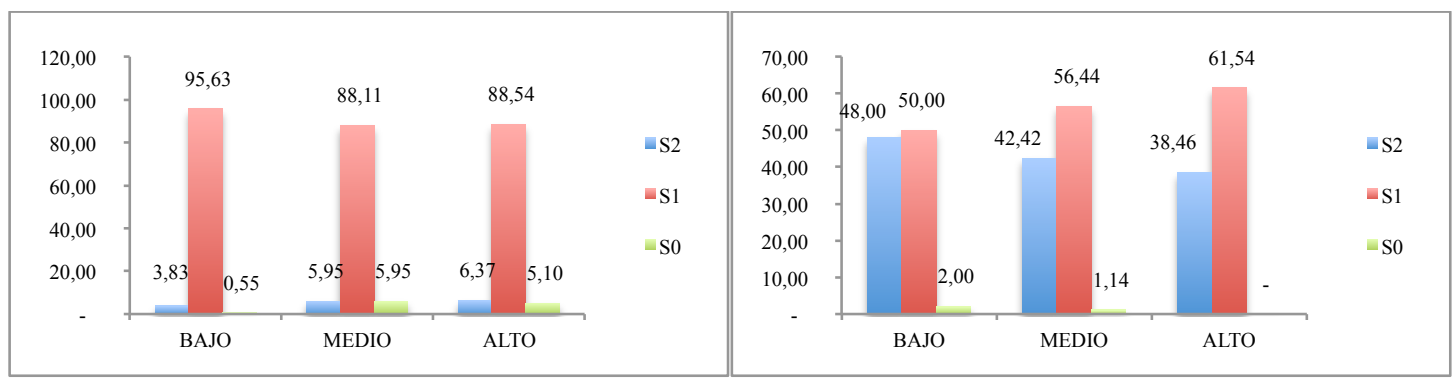

Gráfico 7. Uso de -/s/ en el contexto prevocálico Gráfico 8. Uso de -/s/ en el contexto prevocálico átono según el nivel social. tónico según el nivel social.

Como se observa en el Gráfico 7, en el contexto prevocálico átono la aspiración es la variante habitual (tal como se mencionó en líneas anteriores); no obstante, la 
retención de la sibilante es mayor, de nuevo, cuanto mayor es el nivel social de los individuos.

En cambio, si reparamos en los datos del Gráfico 8 podemos comprobar que, en este caso, el retenimiento del sonido es mayor cuanto menor es el nivel social de los informantes. No obstante, aunque podríamos hablar de un aumento de la consciencia del nivel de prestigio del sonido en los individuos de niveles sociales más bajos, esta tendencia revela, verdaderamente, una acomodación lingüística del hablante cuando une un segmento consonántico, en este caso, fricativo, a la vocal siguiente.

b) El contexto preconsonántico

En la mayor parte de los estudios realizados sobre el habla en Extremadura (Cummins 1974; Zamora Vicente 1979; Salvador Plans 1987; García Mouton 1994; Montero Curiel 1997; González Salgado 2003b) se ha afirmado que, ante consonante, la solución aspirada es la más habitual. ${ }^{16}$

En el habla de Mérida, estas tendencias pueden corroborarse en los siguientes resultados:

\begin{tabular}{lcccccc} 
& /ptk/ & /bdyg/ & /fsh/ & $/ \mathrm{mn} /$ & $/ \overline{\mathrm{f}} /$ & /I \\
\hline Interior & & & & & & \\
S3 & $3,81 \%$ & - & $4,71 \%$ & - & - & $11,90 \%$ \\
S2 & $96,05 \%$ & $100 \%$ & $95,28 \%$ & $100 \%$ & - & $88,09 \%$ \\
S0 & $0,12 \%$ & - & - & - & - & - \\
Final & & & & & & \\
S3 & $1,4 \%$ & $0,59 \%$ & $2,46 \%$ & $0,7 \%$ & - & $2,2 \%$ \\
S2 & $97,32 \%$ & $97,38 \%$ & $96,55 \%$ & $98,76 \%$ & $100 \%$ & $96,68 \%$ \\
S0 & $1,26 \%$ & $1,82 \%$ & $1,25 \%$ & $0,52 \%$ & - & $1,1 \%$ \\
\hline
\end{tabular}

Tabla 7. Distribución de las variantes de -/s/ según el contexto preconsonántico.

Como se observa en la Tabla 7, en posición interior el sonido suele aspirarse de forma habitual, sobre todo ante oclusivas sonoras /bdgy/; las elisiones de -/s/ son poco frecuentes y el mantenimiento de la sibilante aparece, de forma significativa, cuando

\footnotetext{
${ }^{16}$ Navarro Tomás advertía este uso relacionándolo con un cambio que finaliza en la aspiración del sonido y presentándolo como habitual en las hablas meridionales. El autor lo condenaba con las siguientes palabras: "la pronunciación correcta española, aun en su forma menos culta, rechaza esta transformación” (Navarro Tomás 2004: 107).
} 
el segmento se pronuncia ante líquidas (11,90\%), fricativas /fsh/ $(4,71 \%)$ y, finalmente, cuando precede a las oclusivas sordas /ptk/ (3,81\%). No obstante, estos resultados y los correspondientes a la realización de -/s/ en posición preconsonántica, interior y final, revelan que el resultado más común es la aspiración y que, por ello, las variantes utilizadas aparecen con una frecuencia de uso muy escasa.

Si observamos cómo se presentan estas afirmaciones atendiendo al nivel social de los informantes seleccionados para esta investigación, podemos advertir los siguientes resultados:

\begin{tabular}{|c|c|c|c|c|c|c|c|c|c|c|c|}
\hline & \multicolumn{3}{|c|}{ ptck } & \multirow{2}{*}{$\begin{array}{c}\text { bdyg } \\
\text { BAJO-MEDIO- } \\
\text { ALTO }\end{array}$} & \multicolumn{3}{|c|}{ fsh } & \multirow{2}{*}{$\begin{array}{l}\text { mn } \\
\text { BAJO-MEDIO- } \\
\text { ALTO }\end{array}$} & \multicolumn{3}{|c|}{1} \\
\hline & BAJO & MEDIO & ALTO & & BAJO & MEDIO & ALTO & & BAJO & MEDIO & ALTO \\
\hline s3 & 1,23 & 4,28 & 5,29 & & & 3,85 & 16,67 & & 8,33 & 21,05 & \\
\hline s2 & 98,69 & 95,55 & 94,43 & 100 & 100 & 96,15 & 77,78 & 100 & $\begin{array}{c}91,6 \\
7\end{array}$ & 78,95 & 100 \\
\hline so & 0,08 & 0,16 & 0,28 & & & - & 5,56 & & - & - & \\
\hline
\end{tabular}

Tabla 8. Distribución de las variantes de -/s/ según el contexto preconsonántico interior y el nivel social.

Por una parte, si atendemos a los resultados propuestos en el Tabla 8, comprobamos que las diferencias encontradas entre el nivel social y el factor lingüístico se observan en los mismos contextos, esto es, cuando la -/s/ precede a las oclusivas sordas o las fricativas y las líquidas. En esta situación, el uso de la variante estándar siguió las tendencias que se han podido observar hasta ahora en nuestra investigación: cuanto mayor es el nivel social del individuo, mayor es también el uso de S3. Sin embargo, estas afirmaciones únicamente se pueden confirmar en los dos primeros contextos (oclusivas sordas y fricativas); ante líquidas, en cambio, los informantes del nivel medio prefirieron, en un mayor número de realizaciones, usar el sonido estándar $(21,05 \%)$ y, aunque con una menor frecuencia, los del nivel bajo (8,3\%). 


\begin{tabular}{|c|c|c|c|c|c|c|c|c|c|c|c|c|c|c|c|}
\hline & \multicolumn{3}{|c|}{ ptck } & \multicolumn{3}{|c|}{ bdyg } & \multicolumn{3}{|c|}{ fsh } & \multicolumn{3}{|c|}{$m n$} & \multicolumn{3}{|c|}{ I } \\
\hline & BAJO & MEDIO & ALTO & BAJO & MEDIO & ALTO & BAJO & MEDIO & ALTO & BAJO & MEDIO & ALTO & BAJO & MEDIO & ALTO \\
\hline s3 & 0,77 & 0,58 & 5,02 & 0,64 & 0,47 & 4,10 & 2,78 & 1,85 & 4,55 & - & 0,38 & 3,23 & 2,08 & 1,11 & 5,88 \\
\hline s2 & 98,08 & 95,78 & 83,01 & 97,76 & 96,70 & 90,16 & 95,83 & 86,11 & 68,18 & 98,96 & 96,54 & 93,55 & 97,92 & 96,67 & 94,12 \\
\hline so & 1,15 & 3,64 & 11,96 & 1,60 & 2,83 & 5,74 & 1,39 & 12,04 & 27,27 & 1,04 & 3,08 & 3,23 & - & 2,22 & - \\
\hline
\end{tabular}

Tabla 9. Distribución de las variantes de -/s/ según el contexto preconsonántico final y el nivel social.

En posición final, en cambio, los resultados son ciertamente diferentes; de nuevo, la aspiración es la variante habitual pero, según el punto de articulación de la consonante siguiente, los informantes seleccionaron los sonidos S2 o SO. Como muestra el Tabla 9, aunque el retenimiento de -/s/ se observa en escasas realizaciones, es en el nivel alto donde existe un mayor índice de uso, sobre todo ante líquidas $(5,88 \%)$ y oclusivas sordas $(5,01 \%)$ y, posteriormente, ante fricativas $(4,54 \%)$, oclusivas sonoras $(4,09 \%)$ y nasales (3,22\%). En cambio, la elisión del sonido es la variante seleccionada en ciertos contextos, fundamentalmente ante oclusivas sordas $y$ fricativas.

En estos casos se advierte que los individuos del nivel alto prefieren esta variante en un $11,96 \%$ de las realizaciones cuando $-/ \mathrm{s} /$ está delante de las oclusivas sordas; en el contexto preconsonántico fricativo, en cambio, los informantes del nivel medio $(12,03 \%)$ y el nivel alto $(27,7 \%)$ utilizaron So en sus contestaciones.

\section{Conclusiones}

En este trabajo se ha podido comprobar que, en Mérida, es común encontrar variantes de -/s/ que han sido consideradas, tradicionalmente, propias de la zona meridional de la Península esto es, la aspiración y la elisión siendo la primera, no obstante, la más habitual en la localidad. Por tanto, hemos de corroborar que, en este caso, la situación geolingüística del municipio fomenta el uso este tipo de fenómenos. Sin embargo, a lo largo de esta investigación hemos podido advertir que, de igual forma, existen factores lingüísticos y extralingüísticos que influyen, bien en el mantenimiento de la variante estándar o bien en el empleo de realizaciones locales. 
Por una parte comprobamos que, cuando la -/s/ está en posición interior de palabra, la aspiración es la variante habitual pero, en cambio, cuando esta se encuentra en situación final, la elisión es la más utilizada y el mantenimiento del sonido se emplea, además, con una mayor frecuencia. Igualmente, en este análisis hemos verificado que existe una relación significativa entre el nivel social de los individuos y el uso del sonido; así, tanto en posición interior como en final de palabra, cuanto mayor es el nivel social, mayor probabilidad existe de encontrar la variante S2 y menor, por tanto, del uso de la aspiración y la elisión.

También hemos podido advertir que el factor contextual y el social influyen en el uso de las diferentes variantes de -/s/; de esta forma, hemos observado, por una parte, que el contexto prevocálico favorece la retención de la sibilante, principalmente cuando el sonido está situado en posición final de palabra y ante vocal tónica. En este caso, y a diferencia de los resultados anteriores, el mantenimiento de $-/ \mathrm{s} /$ desciende a medida que aumenta el nivel social de los individuos. Ante consonante, de igual forma, hemos observado que, aunque la aspiración sigue siendo el sonido común entre los emeritenses, el lugar que ocupa la -/s/ en la palabra y el tipo de consonante siguiente influyen en la selección de los tipos de $-/ \mathrm{s} /$, fundamentalmente ante los sonidos oclusivos sordos, fricativos y líquidos. Así, en posición interior la -/s/ se mantiene ante estos sonidos, sobre todo en los informantes del nivel alto. No obstante, esta norma no sigue la misma tendencia cuando $-/ \mathrm{s} /$ aparece ante las consonantes líquidas, donde fueron los individuos del nivel medio quienes presentaron un mayor número de realizaciones.

Por tanto, los tres factores que hemos utilizado como punto de partida para conocer la extensión del fenómeno en el habla Mérida, geográficos, lingüísticos y sociales, han demostrado ser fundamentales para comprobar el empleo de las variantes estudiadas y, sobre todo, para observar las peculiaridades del sonido -/s/ en la localidad. 


\section{Referencias}

ALBA, Orlando (1982) Estratificación social del español de Santiago de los Caballeros: la /s/ implosiva, Tesis de licenciatura inédita, Río Piedras: Universidad de Puerto Rico.

ALMEIDA, Manuel (1989) El habla rural en Gran Canaria, La Laguna: Universidad de La Laguna.

Alonso, Dámaso (1973) "Sobre la -s final de sílaba en el mundo hispánico", Obras completas, vol. I, 72-82.

Alvar, M. (1972) Niveles socio-culturales en el habla de Las Palmas de Gran Canaria, Las Palmas de Gran Canaria: Excmo. Cabildo Insular de Gran Canaria.

BLANCO, M. (1997) Aproximación a la cronología de las transformaciones funcionales de las labiales y sibilantes del español, Santiago de Compostela: Universidad de Santiago de Compostela.

CALERO, María Ángeles (1993) Estudio sociológico del habla de Toledo: segmentos fonológicos /s/y/J/, Lleida: Pagés.

Cedergren, Henrietta (1983) "Sociolingüística", en H. López Morales (ed.), Introducción a la lingüística actual, Madrid: Playor, 147-165.

CUMmins, Joan (1974) El habla de Coria y sus cercanías, Londres: Tamesis Books Limited.

FERNÁNDEZ SEVILLA, Julio (1980), “Los fonemas implosivos en español”, Thesaurus, 35, 3, 456505.

García Mouton, Pilar (1994) Lenguas y dialectos de España, Madrid: Arco/Libros.

García Mouton, Pilar \& Moreno FernándeZ, Francisco (1994) “El atlas lingüístico y etnográfico de Castilla-La Mancha. Materiales fonéticos de Ciudad Real y Toledo", Geolingüística. Trabajos Europeos, Madrid: CSIC, 111-153.

GonzÁlez SAlgado, José Antonio (2003a) Cartografía lingüística de Extremadura. Origen y distribución del léxico extremeño, Madrid: Universidad Complutense, [CD-ROM].

GonzÁlez SALGAdo, José Antonio (2003b) "La fonética de las hablas extremeñas", Revista de Estudios Extremeños, LIX, 2, 589-619.

HERASIMCHUCK, Eleanor (1972) "The linguistic dimensions of a bilingual neighborhood", en FISHMAN et al., Bilingualism in the barrio, Bloomington: Indiana University Press, 349464.

KIPARSKY, Paul (1983) Los objetivos de la teoría lingüística, Madrid: Gredos.

LABOV, William (1972) Sociolinguistic Patterns, Philadelphia, Pennsylvania: University of Pennsylvania Press. 
Dialectologia 16 (2016), 93-116.

ISSN: 2013-2247

LIPSKI, John (1983) "Reducción de /s/ en el español de Honduras", Nueva Revista de Filología Hispánica, 32, 273-288.

PILEI (1971) Cuestionario para el estudio coordinado de la norma lingüística culta. Madrid: CSIC.

López MoRAles, Humberto (1983) Estratificación social del español de San Juan de Puerto Rico, México: Universidad Autónoma de México.

Martín Butragueño, Pedro (1995) "La variable (s) en el sur de Madrid. Contribución al estudio de la frontera de las hablas meridionales del español", Anuario de letras, 33, 5-57.

MÉNDEZ DosUNA, Julián (1987) “La aspiración de "s" como proceso condicionado por el contacto de sílabas", Revista Española de Lingüística, 17, 15-35.

Molina MARToS, Isabel (1991) Estudio sociolingüístico de la ciudad de Toledo, Madrid: Universidad Complutense.

Molina MARTOS, Isabel (1998) La fonética de Toledo. Contexto Geográfico y Social, Alcalá de Henares: Universidad de Alcalá.

Montero CURIEL, Pilar (1997) El habla de Madroñera (Cáceres), Cáceres: Universidad de Extremadura.

MoReno FERnÁNDEZ, Francisco (1990) Metodología Sociolingüística, Madrid: Gredos.

MoYa CORRAL, José Antonio (1979) La pronunciación del español de Jaén, Granada: Universidad de Granada.

NAVARRo TOMÁs, TOMÁs (2004) Manual de pronunciación española, Madrid: Centro de Investigaciones Científicas (CSIC).

Salvador Plans, Antonio (1987) "Principales características fonético-fonológicas", en A. Viudas Camarasa, M. Ariza Viguera \& A. Salvador Plans, El habla en Extremadura, Mérida: Editora Regional de Extremadura, 25-37.

SAMPER PADILla, José Antonio (1999) Estudio sociolingüístico del español de las Palmas de Gran Canaria, Las Palmas: Caja de Canarias.

SAMPER PADILLA, José Antonio (2001) "La variación fonológica: los estudios hispánicos sobre la $s$ implosiva", Las normas regionales y socioculturales. La variación lingüística. < accessed July 2014,http://cvc.cervantes.es/obref/congresos/valladolid/ponencias/unidad_diversidad_del_ espanol/1_la_norma_hispanica/samper_j.htm>.

TerReLL, Tracy (1978) “La aspiración y elisión de /s/ en el español porteño, ALM, 16, 41-66.

TeRreLL, Tracy (1979) “Final /s/ in Cuban Spanish", Hispania, 62: 4, 599-612. 
TORREBLANCA, Máximo (1989) "La /s/ implosiva en español: sobre las fechas de su aspiración", Thesaurus, 44 (2), 281-303.

TRUdGilL, Peter \& Juan Manuel Hernández CAMPoY (2007) Diccionario de Sociolingüística, Madrid: Gredos.

VIDA CASTRO, Matilde (2003) Restricciones universales sobre la variación de /-s/ en la distensión silábica. Investigación sobre el español hablado en la ciudad de Málaga, Tesis doctoral. Málaga: Área de Lingüística General de la Universidad de Málaga.

WIDDISON, Kirk (1993) "Hacia los orígenes de la s aspirada en español", Estudios de fonética experimental, vol. 5, 34-59.

ZAMORA VICENTE, Alonso (1943) El habla de Mérida y sus cercanías, Madrid: Anejo XXIX de la Revista de Filología Española.

ZAMORA VICENTE, Alonso (1979) “Hablas de Tránsito", Dialectología española, Madrid: Gredos, 332-336. 